\title{
Absence of nystagmus during REM sleep in patients with vestibular neuritis
}

\author{
I Eisensehr, S Noachtar, M Strupp, H v Lindeiner, T Brandt, U Büttner
}

\begin{abstract}
Saccades, including fast phases of nystagmus, disappear during drowsiness and non-rapid eye movement (NREM) sleep, but are present during the alert state and REM sleep. The purpose of this study was to determine whether spontaneous nystagmus is present in patients with vestibular neuritis during REM sleep.

Eight patients with spontaneous nystagmus due to vestibular neuritis and eight control patients without any nystagmus underwent at least one night of polysomnography. Fast phases of nystagmus were analyzed. The number of right and left horizontal saccades were counted, first during 3-5 minute samples of the awake state before sleep onset, then during the first REM episode and the last REM episode of nocturnal sleep, and finally during the alert state in the morning after nocturnal sleep.
\end{abstract}

All patients with vestibular neuritis showed significantly more saccades (fast phases) towards the side contralateral to their vestibular lesion in the awake state before and after the polysomnography. This reflects their spontaneous nystagmus. By contrast, during REM sleep the patients with vestibular neuritis showed no preponderance in saccade direction. The eye movement pattern in REM was the same for patients and controls.

In conclusion, peripheral vestibular im-

Department of Neurology, Klinikum Grosshadern, Ludwig-Maximilians University,

Marchioninistrasse 15, 81377 Munich,

Germany

I Eisensehr

S Noachtar

M Strupp

$\mathrm{H} v$ Lindeiner

T Brandt

U Büttner

Correspondence to:

Dr I Eisensehr

eisen@

nefo.med.uni-muenchen.de

Received 19 October 2000 and in revised form

9 February 2001

Accepted 26 March 2001 during the awake state in patients with acute vestibular neuritis also occurs during REM sleep.

\section{Methods}

STUDY POPULATION

Informed consent was obtained from all participants in the study. Eight patients with vestibular neuritis and eight controls had a complete physical examination and underwent at least one night of polysomnography (PSG). Polysomnography of the patients with vestibular neuritis was performed from days 1 to 6 after the onset of symptoms of vestibular neuritis (mean 69 hours, range 24-144 hours). For inclusion in the study, nystagmus (slow phase velocity of at least $7 \%$ in the dark) before and after the PSG night had to be present in patients with vestibular neuritis. The diagnosis of vestibular neuritis was based on criteria described in detail elsewhere. ${ }^{67}$

The patients without vestibular neuritis had been referred to the sleep laboratory because of suspected sleep disorders. They were included in the study as controls if they had no history of vertigo and no spontaneous nystagmus during the awake state as verified with Frenzl's glasses.

\section{SLEEP STUDIES}

The recording and scoring of the PSG were performed as described elsewhere. ${ }^{8}$ An electrooculogram (EOG) was obtained from electrodes placed lateral to both eyes and to the infraorbital and supraorbital regions; EOG calibration was performed at the beginning and the end of the PSG and included eye excursion of $35^{\circ}$ from the midline to the right and left, vertical views with and without Frenzl's glasses on, and provocation of vertical and horizontal optokinetic nystagmus. A time constant of 10 seconds was chosen for the EOG. Velocities of the slow phases of horizontal eye movements can be calculated by summarising the amplitudes of the fast phases during a certain time period. Slow phase velocities were exclusively determined for patients with vestibular neuritis during the awake state, when long periods of obvious nystagmus in one direction were present. Right and left (ipsilateral and contralateral) horizontal saccades were counted separately during 3-5 minute samples of the awake state before sleep onset, the awake state after the nocturnal sleep period, and the first and the 
last continuous REM episodes of the nocturnal sleep. Horizontal eye movements were scored only if they had an excursion of $2^{\circ}$ or more and a velocity of at least $50 \%$ second. ${ }^{9}$ Any preponderance in saccade direction was considered potential nystagmus.

\section{DATA PRESENTATION AND STATISTICS}

Data were analyzed using the SPSS statistical package for Windows 9.0. They are presented as mean (SD) if not stated otherwise. The data were tested for normal distribution with the Kolmogorow-Smirnov test. Data points between and within the two study groups were compared using the $\chi^{2}$ test and Mann-Whitney $U$ test for paired and independent samples. In the two groups (controls/vestibular neuritis), the numbers of right/ipsilateral and left/ contralateral horizontal saccades were compared within awake and REM sections to detect directional preponderance of horizontal saccades and nystagmus. A p value $<0.05$ was considered significant.

\section{Results}

Patients with vestibular neuritis were slightly older than controls (vestibular neuritis 65.1(14.7) years, controls 55.4 (19.8) years) and were more often women $(n=7)$ than controls $(n=4),(p>0.05)$. Vestibular neuritis was located on the right in five patients and on the left in three patients. Controls were diagnosed as having periodic limb movement disorder $(n=2)$, narcolepsy $(n=2)$, obstructive sleep apnoea $(n=1)$, and sleep state misperception $(n=3)$. These disorders are not reported to be associated with ocular-motor disorders. As we wanted to score rapid eye movement directions and were not interested in analyzing sleep macrostructure, we thought that our controls were adequate. All patients with vestibular neuritis had spontaneous nystagmus, which was best seen with Frenzl's glasses on, and to a lesser extent during fixation before and after the night of recording. The slow phase velocity with the eyes closed in patients with vestibular neuritis had decreased slightly from the evening before to the morning after the PSG (evening $11.05(6.80) \%$ s; morning 8.39 $(1.32) \%$ s $p>0.05)$.

The videotapes showed that all patients and controls lay on their back without turning their

Table 1 Saccades during wakefulness and REM sleep of patients with vestibular neuritis and control patients

\begin{tabular}{lllll}
\hline & $\begin{array}{l}\text { Number of } \\
\text { saccades/s }\end{array}$ & $\begin{array}{l}\text { Patients with } \\
\text { vestibular } \\
\text { neuritis }\end{array}$ & $\begin{array}{l}\text { Control } \\
\text { patients }\end{array}$ & $\begin{array}{l}\text { P Value } \\
\text { Mann-Whitney } \\
\text { Utest }\end{array}$ \\
\hline Initial wakefulness & $\begin{array}{l}\text { Contralateral/left } \\
\text { Ipsilateral/right }\end{array}$ & $\begin{array}{l}1.1(0.54) \\
0.2(0.21)\end{array}$ & $\begin{array}{l}0.42(0.19) \\
0.38(0.18)\end{array}$ & 0.012 \\
& $\begin{array}{l}\text { Contralateral/left } \\
\text { Last wakefulness }\end{array}$ & $0.79(0.34)$ & $0.38(0.23)$ & 0.012 \\
& Insilateral/right & $0.16(0.10)$ & $0.38(0.24)$ & \\
Initial REM & $\begin{array}{l}\text { Contralateral/left } \\
\text { Last REM }\end{array}$ & $0.21(0.24)$ & $0.13(0.10)$ & 0.236 \\
& Ipsilateral/right & $0.23(0.21)$ & $0.13(0.11)$ & \\
& Contralateral/left & $0.17(0.15)$ & $0.14(0.07)$ & 0.123 \\
& Ipsilateral/right & $0.23(0.17)$ & $0.13(0.05)$ & \\
\hline
\end{tabular}

Contralateral and ipsilateral refers to the side of the vestibular lesion in patients with vestibula neuritis; right and left refers to control patients. Values are given as mean (SD). During REM sleep, none of the patients with vestibular neuritis showed any significant preponderance in saccade direction (Mann-Whitney $U$ test). heads extensively to the right or left side during the analyzed awake and REM periods. All patients with vestibular neuritis showed significantly more saccades to the side contralateral ( $v$ ipsilateral) to their vestibular lesion in the awake state (eyes open and closed), indicating spontaneous nystagmus $(\mathrm{p}=0.012)$ (table 1 ). During REM sleep, none of the patients with vestibular neuritis showed either any nystagmus or any significant preponderance in saccade direction. Similarly, no tonic deviation was found in patients with vestibular neuritis during REM sleep (analyzing eye in head position with EOG and synchronised videotape). No difference in saccade direction was found in control patients during the awake state or REM sleep. Occasionally, brief phases (about 2 seconds long) of nystagmoid jerks (one to four jerks a second) or slow horizontal eye movements with velocities between 10 to $20 \%$ s (fig 1 A) with changing directions and without any directional preponderance were found in both patients and controls during REM sleep. Saccadic eye movements were not found in non-REM (NREM) sleep.

\section{Discussion}

This is the first prospective study comparing eye movements in REM sleep of well defined patients with vestibular neuritis with those of controls. We found that the mechanisms generating REMs during desynchronised sleep are not influenced by mechanisms producing nystagmus in vestibular neuritis. Moreover, the nystagmus associated with vestibular neuritis was abolished during NREM sleep.

Studies investigating eye movements during REM sleep of patients with nystagmus in the awake state due to various CNS lesions report contradictory findings. ${ }^{10-13}$ Gordon et al, ${ }^{10}$ who examined six patients in a vegetative state, and Appenzeller et $a l,{ }^{11}$ who studied three patients with alcoholic and one patient with posttraumatic encephalopathy, described brief phases of nystagmoid jerks during REM sleep. These jerks differed from the continuous nystagmus seen during wakefulness (no description of nystagmus direction). However, brief phases of irregular nystagmoid jerks during REM sleep have also been described in healthy subjects, ${ }^{13}$ and we also found nonsystematic brief phases of nystagmoid jerks in vestibular neuritis and controls. These may be related to a gaze holding deficit on lateral gaze, which might correspond to a neuronal integrator disorder during REM sleep. By contrast, two studies reported that the nystagmus generated by CNS lesions in the awake state disappears during NREM and REM sleep. ${ }^{12}{ }^{13}$ Another study found a nearly total suppression of rapid eye movements directed away from the side of the lesion during REM sleep in six patients with unilateral attentional neglect, ${ }^{14}$ although phases of nystagmus were not described in the study.

In cats, unilateral labyrinthectomy and unilateral section of the eighth nerve resulted in spontaneous nystagmus during wakefulness for 


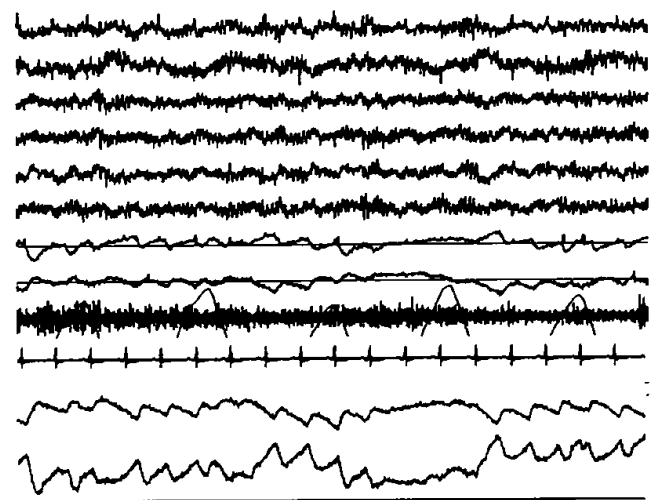

B
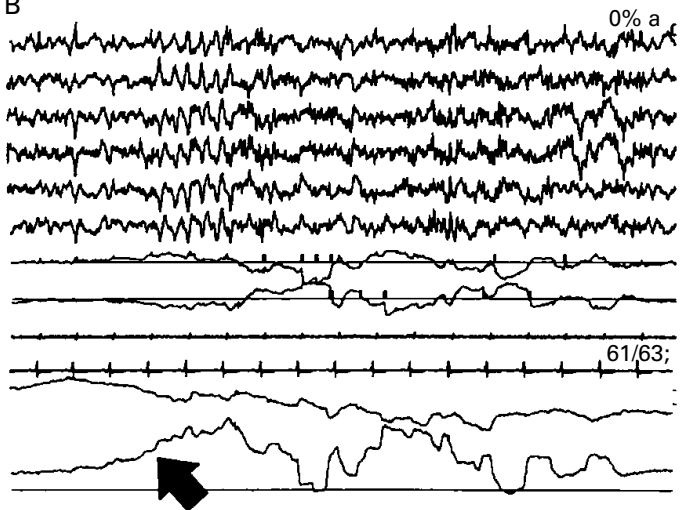

Vertical eye movement Horizontal eye movement

C4-A1

C3-A2

$\mathrm{O} 2-\mathrm{Cz}$

$\mathrm{O} 1-\mathrm{Cz}$

$\mathrm{T} 4-\mathrm{Cz}$

$\mathrm{T} 3-\mathrm{Cz}$

LBE-A1

RAE-A2

EMG chin

ECG

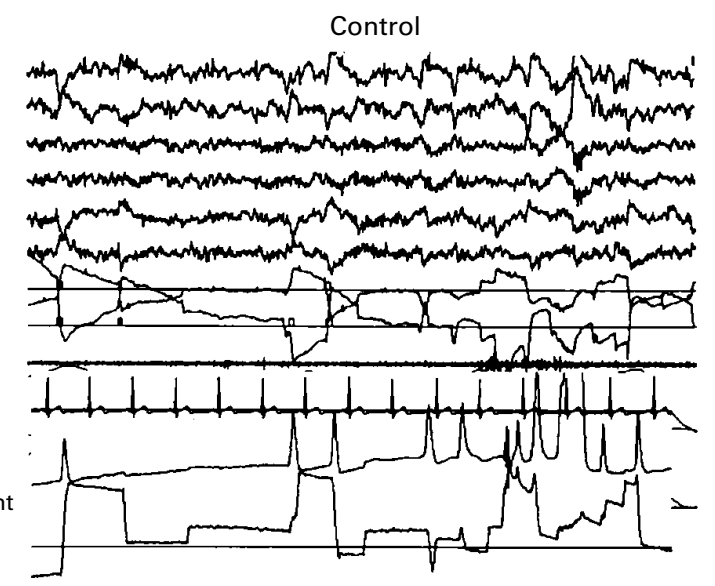

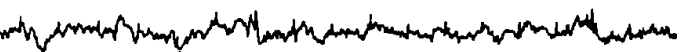

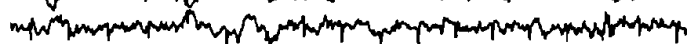
(2)

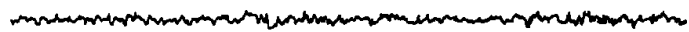

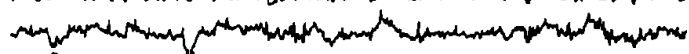

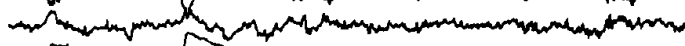
 Ln+

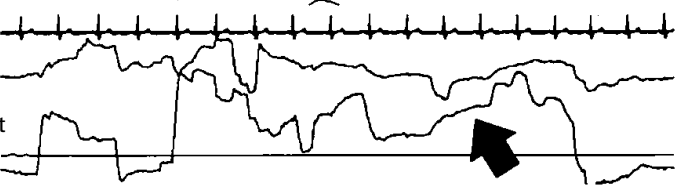

$5 \mathrm{~s}$

Figure 1 (A) Thirty second sample of the awake state before sleep onset of the polysomnogram for a patient with right vestibular neuritis and one control patient. The control patient shows several blinking artifacts in the channel for the vertical eye movements. To get highly artefact free awake phases the patients and controls were told to relax as much as possible during the initial and final wake phases. This might have been easier for controls than for patients with vestibular neuritis, who mostly complained about nausea when they were awake. Therefore, the low chin muscle tone in the control indicates good relaxation by contrast with the patients with vestibular neuritis. (B) Thirty second sample of the first REM period of the polysomnogram for a patient with right vestibular neuritis and one control patient. The drift of slow eye movements to the right, which preceded a series of rapid eye movements in vestibular neuritis, was not a frequent finding. Such an occasional drift occurred in various directions and was also seen in controls. A1=left ear; A2=right ear; $L B E=$ left below eye; $R A E=$ right above eye; $E C G=$ electrocardiogram.

several days, ${ }^{15}{ }^{16}$ which was largely depressed or completely abolished during NREM and REM sleep.

Studies on the influence of vestibular stimulation on eye movements during sleep in normal subjects have given contradictory results. ${ }^{17-19}$ Reding and Fernandez, ${ }^{17}$ using perrotatory stimulation at 10 minute intervals in a sample of five children, found that nystagmic jerks (direction not specified) were significantly associated with the occurrence of rapid eye movements in REM sleep. However, brief phases of nystagmic jerks during REM sleep have also been described in healthy subjects under normal conditions. ${ }^{13}$ Other studies in adult humans demonstrated that neither postrotatory nor perrotatory vestibular stimulation elicited nystagmus during any stage of sleep. ${ }^{18} 19$ However, slow compensatory conjugate deviation of the eyes occurred with each oscillation during REM sleep, whereas rapid eye movements were absent in the study of Tauber $\mathrm{et}^{\mathrm{al}} \mathrm{l}^{18}$; this suggests some vestibular influence on eye movements during REM sleep. We exclusively evaluated the number and direction of horizontal saccades during REM sleep. Therefore we cannot exclude vestibular influence on other characteristics of rapid eye movements during REM sleep (number of clusters versus single rapid eye movements, velocities etc). However, nystagmus seen during wakefulness in our patients with vestibular neuritis could definitely not be seen during REM sleep. In analogy to the de-efferentation of the motor output during REM sleep, it might be hypothesised that there is a deafferentation of the peripheral vestibular input to the REM sleep generating areas. This deafferentation might originate from REM sleep generating structures in the paramedian pontine reticular formation and inhibit peripheral input to the medial vestibular nucleus, which plays an executive part in rapid eye movements during REM sleep. ${ }^{4520}$ Caloric labyrinthine stimulation during REM sleep should be performed in humans to test this hypothesis.

1 Horn AKE, Büttner-Ennever JA, Büttner U. Saccadic premotor neurons in the brainstem: functional neuroanatomy and clinical implications. Neuroophthalmology 1996;16:229-40.

2 Hoshino K, Pampeiano O, Magherini PC, et al. The oscillatory system responsible for the oculomotor activity during
the bursts of REM. Arch Ital Biol 1976;114:278-309.

3 Kamogawa H, Ohki Y, Shimazu H, et al. Inhibitory input to pause neurons from pontine burst neuron area in the cat. Neurosci Lett 1996;203:163-6. 
4 Bizzi E, Pompeiano O, Somogyi I. Vestibular nuclei: activity of single neurons during natural sleep and wakefulness. 64;145:414-15.

5 Bizzi E, Pompeiano O, Somogyi I. Spontaneous activity of single vestibular neurons of unrestrained cats during sleep and wakefulness. Arch ital Biol 1964;102:308-30.

6 Strupp M, Arbusow V, Maag KP, et al. Vestibular exercises improve central vestibulospinal compensation after vestibular neuritis. Neurology 1998;51:838-44.

7 Strupp M, Brandt T. Vestibular neuritis. Adv Otorhinolaryngol 1999;55:111-36.

8 Eisensehr I, Ehrenberg BL, Noachtar S, et al. Association of obstructive sleep apnea syndome with plasma epinephrine levels, platelet activation and blood pressure. Neurology 1998;51:188-95.

9 Neetens A, van den Ende P. Eye movements during sleep. Bull Soc Belge Ophtalmol 1989;237:227-44.

10 Gordon CR, Oksenberg A. Spontaneous nystagmus across the sleep-wake cycle in vegetative state patients. Electroenthe sleep-wake cycle in vegetative state patien
cephalogr Clin Neurophysiol 1993;86:132-7.

11 Appenzeller O, Fischer AP. Disturbances of rapid eye movements during sleep in patients with lesions of the nervous ments during sleep in patients with lesions of the nervous

12 Tauber ES, Weitzman ED, Herman J, et al. Absence of nystagmus during REM sleep in a patient with waking nystagmus and oscillopsia. $\mathcal{F}$ Neurol Neurosurg Psychiatry 1973;36: $833-8$
13 Kawahara R, Hazama H, Fukuhara T, et al. Nystagmus and REM density during sleep in patients with brain stem lesions. Waking Sleeping 1980;4:205-10.

14 Doricchi F, Guariglia C, Paolucci S, et al. Disturbances of rapid eye movements (REMs) of REM sleep in patients with unilateral attentional neglect: clue for the understanding of the functional meaning for REMs. Electroencephalogr Clin Neurophysiol 1993;87:105-16.

15 Baldissera F, Broggi G, Mancia M. Nystagmus induced by unilateral labyrinthectomy affected by sleep-wakefulness cycle. Nature 1967;215:62-3.

16 Lenzi GL, Pompeiano O. Orthodromic transmission of 8th nerve volleys through the vestibular nuclei during sleep. Adv Otorhinolaryngol 1970;17:2-27.

17 Reding GR, Fernandez CO. Effects of vestibular stimulation during sleep. Electroencephalogr Clin Neurophysiol 1968;24: $75-9$.

18 Tauber ES, Handelman G, Handelman R, et al. Vestibular simulation during sleep in young adults. Arch Neurol 1972; simulation

19 Jones GM, Sugie N. Vestibulo-ocular responses in man during sleep. Electroencephalogr Clin Neurophysiol 1972;32:43ing

20 Pompeiano O, Morrison AR. Vestibular influences during sleep. I. Abolition of rapid eye movements during desynchronized sleep following vestibular lesions. Arch Ital Biol 1965;103:569-95. 